\title{
Dynamics of sonoluminescing bubbles within a liquid hammer device
}

\author{
Raúl Urteaga, Pablo Luis García-Martínez, and Fabián J. Bonetto \\ Laboratorio de Cavitación y Biotecnología 8400, Instituto Balseiro/Centro Atómico Bariloche, RN, Argentina
}

(Received 29 December 2007; revised manuscript received 7 August 2008; published 14 January 2009)

\begin{abstract}
We studied the dynamics of a single sonoluminescing bubble (SBSL) in a liquid hammer device. In particular, we investigated the phosphoric acid-xenon system, in which pulses up to four orders of magnitude brighter than SBSL in water systems (about $10^{12}$ photons per pulse) have been previously reported [Chakravarty et al., Phys. Rev. E 69, 066317 (2004)]. We used stroboscopic photography and a Mie scattering technique in order to measure the radius evolution of the bubbles. Under adequate conditions we may position a bubble at the bottom of the tube (cavity) and a second bubble trapped at the middle of the tube (upper bubble). During its collapse, the cavity produces the compression of the liquid column. This compression drives impulsively the dynamics of the upper bubble. Our measurements reveal that the observed light emissions produced by the upper bubble are generated at its second collapse. We employed a simple numerical model to investigate the conditions that occur during the upper bubble collapse. We found good agreement between numerical and experimental values for the light intensity (fluence) and light pulse widths. Results from the model show that the light emission is increased mainly due to an increase in noble gas ambient radius and not because the maximum temperature increases. Even for the brightest pulses obtained $\left(2 \times 10^{13}\right.$ photons, about $20 \mathrm{~W}$ of peak power) the maximum temperatures computed for the upper bubble are always lower than $20000 \mathrm{~K}$.
\end{abstract}

DOI: 10.1103/PhysRevE.79.016306

PACS number(s): 78.60.Mq

\section{INTRODUCTION}

Single-bubble sonoluminescence (SBSL) have captivated the attention of the scientific community since its discovery in 1989 [1]. It occurs when an acoustically trapped and periodically driven gas bubble collapses so strongly that the energy focusing at its collapse leads to light emission [2]. The astonishing energy focusing capability that can be achieved, is probably the most exciting feature of this phenomenon. The upscaling of the light pulses intensities and bubble maximum temperatures during the main bubble collapse have been subject of study for many years $[3,4]$. As pointed out by Toegel et al. [5], when the bubble is immersed in water, the water vapor present inside the bubble can prevent this upscaling due to endothermic reactions. Using sulfuric acid, which has a lower vapor pressure and a higher viscosity, Flannigan et al. [6] measured light pulses about two orders of magnitude more intense than previously reported using water. Later calculations of bubble stability indicated that such an intensity increase can be achieved due to an enhanced region of stability that allows larger bubbles and more intense driving pressure, besides the lower amount of vapor present in the bubble [7]. In this case, the position stability (Bjerknes force) limits the maximum driving pressure that can be applied [8]. This limitation may be shifted by including higher harmonics in the excitation producing up to a fourfold increase in light emission (about $0.1 \mathrm{~W}$ peak power) [9]. Probably the easiest way to try to achieve a significant increase in energy concentration is to reduce the excitation frequency [3]. Doing this, the bubble achieves a larger radius and more mechanical energy can be transferred from the acoustic field to the bubble. In the past, the size of the resonator became a practical problem when researchers attempted to use low excitation frequencies. For example in order to achieve a $7 \mathrm{kHz}$ working frequency, a 61 spherical resonator must be used [5]. Stable SBSL inside a liquid hammer tube seems to have the potential to solve the size limitation because it keeps the total volume of the tube small. Pioneer work by $\mathrm{Su}$ et al. [10] used 10 torr of xenon (Xe) dissolved in cooled water in a $50 \mathrm{~cm}$ in length column water hammer tube driven at $10 \mathrm{~Hz}$. They obtained light pulses of $10^{8}$ photons, 10 times greater than previously reported SBSL pulses in water-xenon using resonators systems, reaching a peak power of $0.4 \mathrm{~W}$. Chakravarty et al. [11] tested several fluids with low vapor pressure and high viscosity in a speaker-driven liquid hammer tube using also xenon as the noble gas. They simultaneously rotated the tube along its axis in order to confine the bubbles at the rotation axis (tube centerline) and obtained repetitive light pulses containing $10^{12}$ photons approximately and $1.2 \mathrm{~W}$ peak power. The liquid employed in this case was phosphoric acid in a $16 \mathrm{~cm}$ long liquid hammer tube driven at $35 \mathrm{~Hz}$. In their work, Chakravarty et al. did not investigate the mechanisms that are responsible for the production of such high intensity pulses. In this work, we focused our attention on the liquid hammer dynamics for the phosphoric acid-xenon system. We studied the mechanisms that cause the light emissions using a setup similar to the one used by Chakravarty et al. [11]. We introduced changes to improve stability and reproducibility. The rest of the paper is organized as follows. In Sec. II, we describe the experimental setup. Experimental observations and discussion are presented in Sec. III. In Sec. IV a simple numerical model is used to obtain a better understanding of the observed experimental results. Finally, our conclusions are summarized in Sec. V.

\section{EXPERIMENTAL SETUP}

We show schematics of the experiment in Fig. 1. The system is similar to the one used in Ref. [11] with three main 


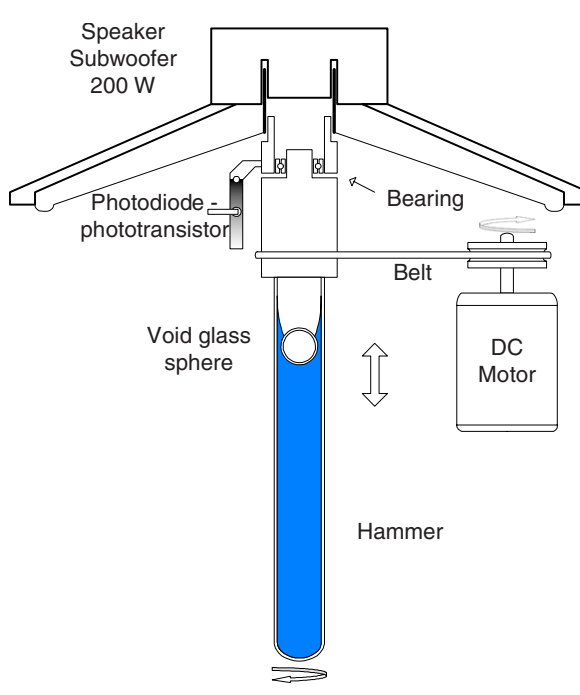

FIG. 1. (Color online) Apparatus employed to drive the liquid hammer tube. A subwoofer speaker generates the vibration and a motor with a belt imposes rotation of the tube about its symmetry axis.

differences. In the first place, we put the excitation speaker on top. We hold the tube from the uppermost position instead of doing this from the lowermost position. In this way, the bottom of the tube is completely visible. The second difference is the mechanism that produces the tube rotation. An external electrical dc motor is coupled to the tube through a belt minimizing the oscillatory mass and reducing the axial play of the system. The third difference is that we use a void glass sphere inside the tube, which is not attached to the tube. This sphere acts as a buoy that prevents the sloshing at the free surface. In addition, the sphere limits the size of the meniscus allowing a wider range of rotation frequency. These modifications do not change the physics we are interest in while enhancing the stability and the range of working frequencies of the system.

A gradual filter is attached to the support of the tube in order to measure the tube vertical position. We use a photodiode phototransistor pair fixed to the table, which produces a signal proportional to the vertical displacement of the tube. On this system, we study the dynamics of the lower cavity and the upper bubbles using two different techniques. Cavity evolution is measured using stroboscopic photographs. A short light flash (about $5 \mu \mathrm{s}$ ) illuminates the cavity while in the opposite side of the tube a CCD camera captures the images (backlighting). Setting the flash frequency slightly lower than the vertical excitation frequency we obtain a sequence of photographs with increasing time delay. In this way, taking only one photograph per cycle we can measure the complete evolution at an equivalent sample frequency determined by $f_{\text {eq }}=f / \Delta f$, where $f$ is the excitation frequency and $\Delta f$ is the frequency difference between the excitation and illumination. This technique is adequate for systems whose dynamics is periodic, i.e., repetitive. Only the light pulse width and the stability (repetitiveness) of the system limit the equivalent time resolution [9]. The upper bubble evolution is determined using stroboscopic photography and the Mie scattering technique. The Mie scattering technique is

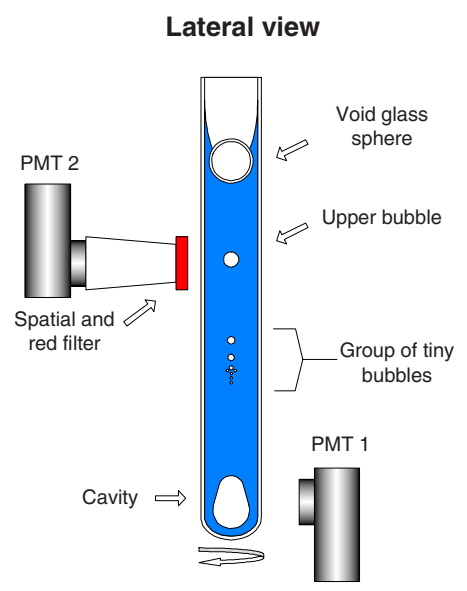

Upper view

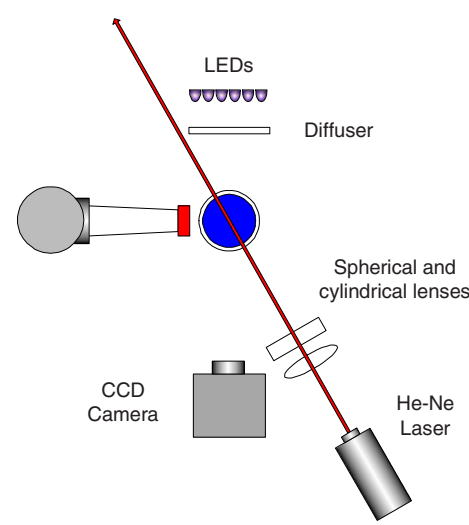

FIG. 2. (Color online) Experimental setup. Two techniques are used to measure the radius evolution of the bubbles. The wellknown Mie scattering and stroboscopic photography. A photomultiplier (PMT 1), placed at the bottom, produces a trigger signal from the light pulse emitted at the cavity collapse. A second PMT equipped with spatial and red filters measures the light scattered by the bubble when it is illuminated with a laser.

extensively used in sonoluminescence $[12,13]$. When the bubble is illuminated by a laser beam, the light scattered by the bubble can be related to the square of its radius if a correct angle of incidence is chosen. A photomultiplier module Hamamatsu $H 957$ placed at $70^{\circ}$ forward scattering measures the light dispersed from the bubble illuminated with a $30 \mathrm{~mW}$ He-Ne laser (PMT2 in Fig. 2). We use a pair of cylindrical and spherical lenses to generate a plane of uniform illumination over the region of movement of the bubble. A spatial filter is used to measure only the light dispersed by the bubble located in the uppermost position, i.e., the bubble that is closer to the free surface (upper bubble in Fig. 2). We use a red filter to prevent saturation with the light pulse emitted by the bubble. Another PMT is used to measure the light pulse emitted from the cavity (PMT1 in Fig. 2). This signal is used as a trigger (zero time scale) when we measure the upper bubble evolution. The Mie scattering technique provides good time resolution for the dynamics (100 ns) and allows us to obtain the complete evolution in one cycle. However, an absolute determination of the radius becomes difficult. On the other hand, the stroboscopic pho- 
tography has time resolution limitations, mainly imposed by the stability of the system, but it gives accurate absolute radius determinations. Combining both techniques, we are able to obtain the complete dynamics of the upper bubble radius with high temporal resolution and accurate absolute values. We measure the intensity of the light emissions from the upper bubble employing the same CCD camera used to take the stroboscopic photographs (with backlighting turned off) integrating light over one period. The camera is slightly out of focus in order to prevent saturation and enhance the dynamical range. This camera also provides the information of the position of the bubble within the tube.

We use a glass tube partially filled with dehydrated phosphoric acid $(\sim 102 \%$, which has a viscosity $\mu=0.25 \mathrm{~Pa} \cdot \mathrm{s})$ doped with xenon gas to a pressure of around 27 mbar. The length of the tube employed is $20 \mathrm{~cm}$, its inner diameter is $12 \mathrm{~mm}$. The liquid column occupies $12 \mathrm{~cm}$ of the glass tube (when it is rotating). Subwoofer excitation frequency is set at $16.55 \mathrm{~Hz}$ with peak-to-peak amplitude of $8 \mathrm{~mm}$. The tube rotates at $20 \mathrm{~Hz}$.

\section{EXPERIMENTAL RESULTS}

The results obtained using the experimental techniques described in Sec. II are presented and discussed in this section. We begin with a brief description of the evolution of the bubbles inside the liquid hammer device during a typical run of the experiment. Starting with the hammer tube at rest, a vertical oscillation is imposed. If the oscillation amplitude is high enough to produce negative pressure in the liquid, a cavitation bubble is formed at some point in the liquid column. Generally, this cavitation bubble appears at a nucleation point on the wall, near the tube bottom. As soon as the bubble becomes visible, a characteristic cavitation noise becomes audible. Because of the rotation, the bubble migrates to the rotation axis while moving down slowly until it reaches the bottom of the tube. This bubble remains there during the whole experiment and produces visible pulses of light in temporal coincidence with each sound beat. We call this bubble the lower bubble or simply the cavity. In the proximity of the cavity we can observe small bubbles that are generated at each collapse of the cavity. These bubbles may combine to form larger ones and they attract each other creating small clusters. These clusters evolve over time and bubbles increase their size rising slowly towards the free surface on top. A particular spatial distribution of the bubbles along the rotation axis is observed, since they are arranged monotonically by increasing size from tube bottom to top. After some seconds, the largest bubble, which is the closest to the free surface, begins to emit light pulses (upper bubble in Fig. 2). Occasionally, two or more bubbles in the column emit light at the same time but the largest (i.e., the upper bubble) is always the brightest. Measurements of the position relative to the free surface [Fig. 3(a)], the number of photons emitted per pulse [Fig. 3(b)], and the pulses full width at half maximum [Fig. 3(c)] for the upper bubble are shown in Fig. 3.

The upper bubble rises due to buoyant force while increasing light emissions until the pressure field present in the

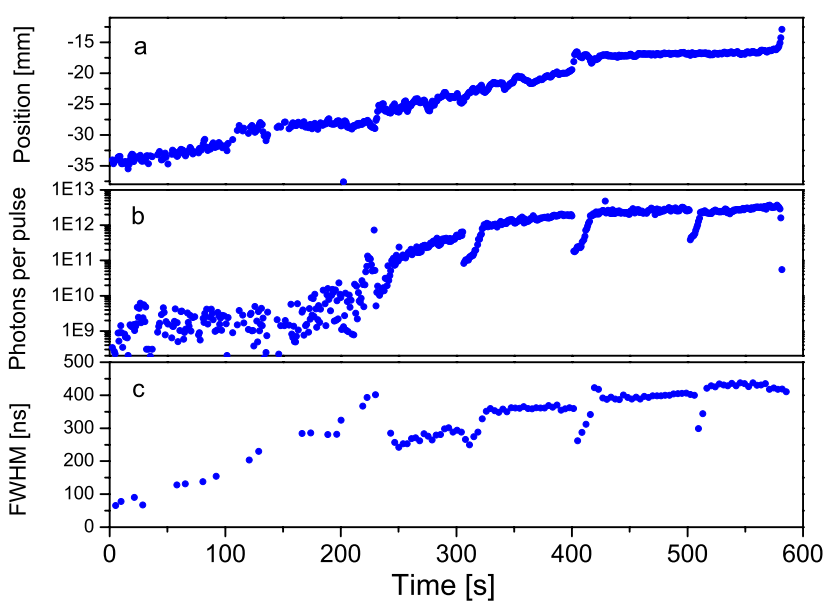

FIG. 3. (Color online) Temporal evolution of the bubble position in the vertical direction from free surface (top), number of photons per pulse (center), and the full-width at half maximum (bottom) of the emitted light pulse. Experiment run with phosphoric acid at $102 \%$, xenon head pressure of $27 \mathrm{mbar}, 16.55 \mathrm{~Hz}$ of excitation frequency, peak-to-peak displacement of $8 \mathrm{~mm}$, and a $28{ }^{\circ} \mathrm{C}$ system temperature.

liquid column traps the bubble balancing the effect of buoyant force $[\sim 420 \mathrm{~s}$ in Fig. 3(a)]. As a result, the bubble position remains constant while the pulse duration and the photons emitted per pulse increase more slowly, showing discontinuities in their evolution [Figs. 3(b) and 3(c)]. Such behavior of the light emission may be explained in terms of increasing bubble gas content, due to rectified diffusion (continuous grow) and bubble coalescence (discontinuities). Since the liquid is saturated in the noble gas, bubbles are not diffusively stable which implies that the rectified diffusion mechanism produces the continuous growth of the bubble [14]. Furthermore, the gas inventory inside the upper bubble can be increased by coalescence with bubbles that come from lower positions in the column [discontinuities in curves of Figs. 3(b) and 3(c)]. When buoyant force becomes dominant (about $580 \mathrm{~s}$ in Fig. 3), the bubble ceases to glow, disappears at the free surface and is replaced by the following bubble in the column which repeats the sequence. The complete cycle duration depends on the driving amplitude (constant during the whole evolution) and takes between 2 and $15 \mathrm{~min}$.

Finally we want to mention some qualitative aspects of the upper bubble evolution which turn out when the tube oscillation amplitude is varied. We observed that the time the bubble spends at the equilibrium position $(t=420 \mathrm{~s}$ to $t$ $=580 \mathrm{~s}$ in Fig. 3) can be enhanced by slightly increasing the tube oscillation amplitude when the bubble begins to depart from this point (near $580 \mathrm{~s}$ in Fig. 3). At this moment, a slight excitation amplitude increase restores the bubble to its equilibrium position (instead of rising until the free surface) and the bubble evolution continues in a similar fashion to the one showed in the last part of Fig. 3. After some time the bubble again tends to rise, but it can again be restored by another slight oscillation amplitude increase and so on. This process was repeated during almost one hour but we did not explore the maximum time that can be achieved. We note 


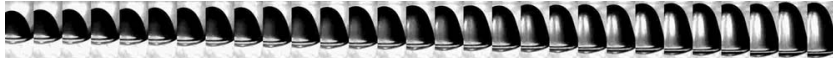

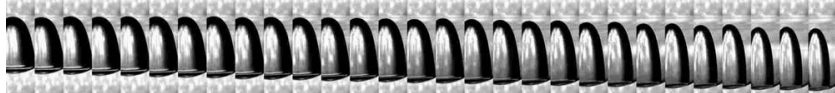

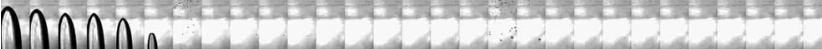

FIG. 4. Sequence of images for the cavity evolution. The tube is rotating at $w=20 \mathrm{~Hz}$ while the vertical excitation is at $16.55 \mathrm{~Hz}$. The equivalent time delay between photographs is $315 \mu \mathrm{s}$. Individual image height is $15.5 \mathrm{~mm}$. The complete sequence shows almost one half of the excitation period.

that if the final oscillation amplitude level reached using this gradual process is applied suddenly (i.e., giving no chances to the bubble for gas incorporation), the bubble significantly diminishes its light emission or even ceases to glow. Thus we referred to a slight amplitude increase as one high enough as to prevent the bubble from rising until the free surface but low enough as to not significantly reduce light emission. Results from numerical computations presented in Sec. IV agree with these two observations namely, that a sudden amplitude increase diminishes light emission and that bigger bubbles (larger $R_{0}$ ) allow higher levels of excitation leading to more light production (inset of Fig. 10).

\section{A. Lower bubble or cavity}

Now we present measurements taken on the lower bubble or cavity located at the bottom of the tube. A sequence of photographs taken at the bottom of the tube during the cavity collapse is shown in Fig. 4.

The almost empty cavity is seen to undergo a relatively slow expansion and a violent main collapse followed by at least two (identifiable) rebounds. The amount of gas contained in the cavity can be determined by stopping the tube excitation and measuring the radius of the bubble that rises from the bottom due to the buoyant force. The radius of that bubble is found to be $170 \pm 20 \mu \mathrm{m}$, approximately constant during the whole evolution of the system. Detailed observation of the main and the second collapse reveals the presence of small gas bubbles released from the cavity, seen as small dark points in Fig. 4 (frames number 7 and 18 of last row). This gas ejection may be regarded as the mechanism responsible for maintaining the gas inventory within the cavity. Assuming axial symmetry and taking into account refraction effects at the tube glass, it is possible to calculate the volume of the cavity at each image of Fig. 4. Results of such procedure are shown in Fig. 5(a), along with the radius of a spherical bubble with the same volume and the tube position, Fig. 5(b).

From cavity volume evolution it is possible to obtain liquid column velocity (i.e., free surface velocity) simply dividing by tube cross section (assuming liquid incompressibility). Linearly fitting the last twelve experimental points before the main collapse in Fig. 5(a) we obtain a velocity of $0.24 \mathrm{~m} / \mathrm{s}$. The pressure increase at the bottom of the tube due to sudden detention of the liquid (water hammer effect) can be estimated as $\Delta p=v \rho c \sim 6$ bar (using $\rho=1800 \mathrm{~kg} / \mathrm{m}^{3}$
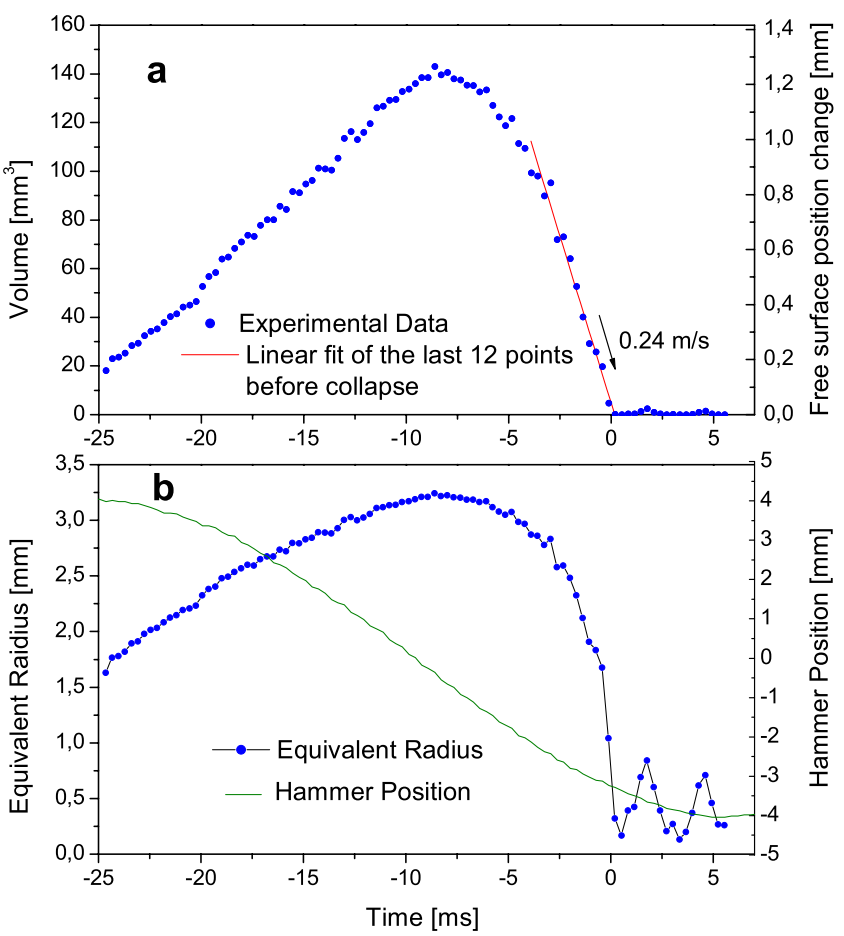

FIG. 5. (Color online) (a) Cavity volume calculated from images of Fig. 4. Linear fit of points preceding main collapse is traced. (b) Radius of an equivalent spherical bubble (left axis) and tube position (right axis).

and $c=1400 \mathrm{~m} / \mathrm{s})$. As this value is two orders of magnitude higher than the gas tube static pressure $(0.027$ bar) we expect pressure disturbances arising from water hammer effect to be the primary excitation that drive the upper bubbles dynamics. In Fig. 5(b) the evolution of the radius of a bubble with the same volume than the cavity is shown. There the rebounds following the main collapse are more evident and, as it is discussed below, observations of the upper bubble show that pressure waves launched at the second cavity collapse (at about $3 \mathrm{~ms}$ in Fig. 5) play a key role in light emission from the upper bubble.

\section{B. Upper bubble}

The measurements for the upper bubble presented in this section were performed when this bubble was placed at $25 \mathrm{~mm}$ below the free surface, which corresponds to a time approximately equal to $260 \mathrm{~s}$ in Fig. 3. Images obtained for the upper sonoluminescing bubble after removing refraction effects at the tube glass are presented in Fig. 6. They show that the bubble keeps its spherical shape in spite of liquid rotation.

Bubble evolution exhibited in Fig. 6 begins with a relatively slow compression followed by a series of expansions and subsequent collapses of decreasing amplitude. Since exposure time of these photographs is equal to one excitation period (about $60 \mathrm{~ms}$ ), all of them show the bright flash emitted by the bubble in that cycle. This flash appears as a white point initially positioned below the bubble (Fig. 6) and indicates the position at which the bubble emits light in that 


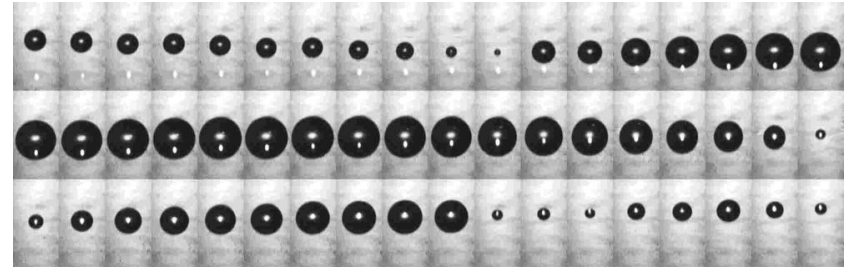

FIG. 6. Sequence of images for the upper stable bubble. Equivalent time delay between frames is $129.3 \mu$ s and individual image height corresponds to $3.5 \mathrm{~mm}$. Refraction effects at the tube wall were removed from these images. The light pulse emitted by the bubble is initially positioned below the bubble image. The central part of the bubble is always illuminated with light coming from backlighting which was not dispersed by the bubble.

cycle. Note that the center of the bubble is always illuminated because of backlighting. These photographs reveal that the emission takes place during the second collapse of the bubble. This result (confirmed by photomultiplier measures when applying Mie scattering technique) may sound unexpected since driving pressure arising from the impact of the liquid column against the tube should be much more intense at the main collapse of the cavity than at the second one [see Fig. 5(b)]. Collecting the light scattered by the bubble (Mie scattering) allows a higher temporal resolution (100 ns) in radius determination than photographs, but it fails in giving reliable absolute values. Combining both techniques one can achieve temporal resolution while obtaining correct absolute values suitable for calibration. These methods were not applied simultaneously because they have different lighting requirements. Figure 7 shows the results of both techniques, taking photographs first and performing Mie scattering light collection immediately afterwards.

Results obtained for the bubble radius evolution from both methods present good agreement. The light pulse coming from the bubble has been suppressed using a low-pass filter $(650 \mathrm{~nm})$. Time equal zero in the graph of Fig. 7 is made coincident with the cavity main collapse whose exact time is determined using a second photo-multiplier placed at

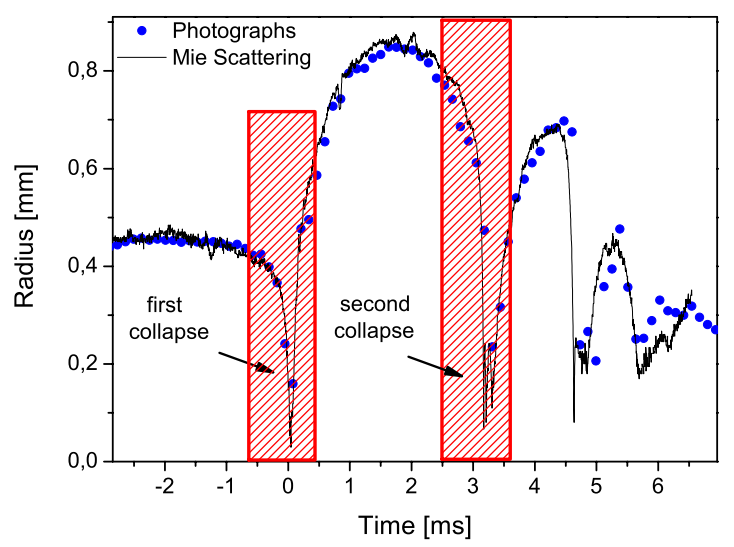

FIG. 7. (Color online) Upper bubble radius obtained using Mie scattering technique (solid). Superimposed dots are values coming from photographs taken immediately before the Mie scattering measurement. A red filter was used in order to prevent the SL pulse at second collapse. the bottom of the tube detecting the cavity light pulse (PMT 1 in Fig. 2). It is observed that the upper bubble first collapse occurs approximately at the same time that the cavity main collapse. Furthermore, it is also observed that the two subsequent rebounds are synchronized with the cavity rebounds [see Fig. 5(b)]. These rebounds are separated by decreasing time intervals lasting some milliseconds. Since bubble response time is much shorter [16] we conclude that upper bubble is driven impulsively each time that the cavity collapses or, equivalently, each time that the liquid column hits the tube. In the next section we analyze in detail the first and the second collapse of the upper bubble induced by the pressure disturbances generated by the cavity collapses at the bottom of the tube.

\section{ANALYISIS}

\section{A. Numerical model}

In this section we analyze in detail the first and second collapses of the upper bubble using Mie scattering measurements and a numerical model for the bubble radius dynamics and the light emission during bubble collapse. The model for the radius temporal evolution to compute the conditions inside the bubble (temperature, pressure, etc.) follows the work from Toegel et al. [5]. In our work we neglect the presence of liquid (mainly water) vapor inside the bubble. This simplification is justified based on the fact that the phosphoric acid has a very low vapor pressure at room temperature. This vapor pressure is at least 2000 times lower than the vapor pressure in pure water. We also neglected mass diffusion of the xenon dissolved in the liquid to the bubble interior and the mass diffusion from the xenon inside the bubble to the liquid. As a consequence of these two assumptions (no vapor inside the bubble and no diffusion of xenon neither in nor out of the bubble) the bubble had pure xenon inside and the bubble mass was constant in time. Once radius, temperature and density are determined, the light emission is computed following the finite opacity model from Hilgenfeldt et al. [15]. Results from that work [15] were reproduced in order to check the code and consistency between models. The heat transfer model in Togel et al. [5] (which was used in this work) is not the same than the one used by Hilgenfeldt et al. Thus, we expected to have some differences in the light emissions computed by Hilgenfeldt and our computations. We obtained maximum discrepancies of $10 \%$ in FWHM and considered them to be acceptable since this parameter is very sensitive to the heat transfer model. The models mentioned above were developed in the context of SBSL using resonators where the driving pressure is sinusoidal $[\mathrm{Pa}$ term of Eq. (1) in Ref. [5]]. In contrast, bubbles inside a liquid hammer are subjected to pressure impulses coming from the liquid plug sudden stop (cavity collapse) whose details are not available, preventing us from the possibility to compute the bubble evolution using the RP model. Nevertheless, since bubble radius evolution is available from experimental determinations we solved the inverse problem, i.e., we obtained the driving pressure from the radius evolution. The result of the computation will bring some information about the details of the compression of the liquid column when the cavity 


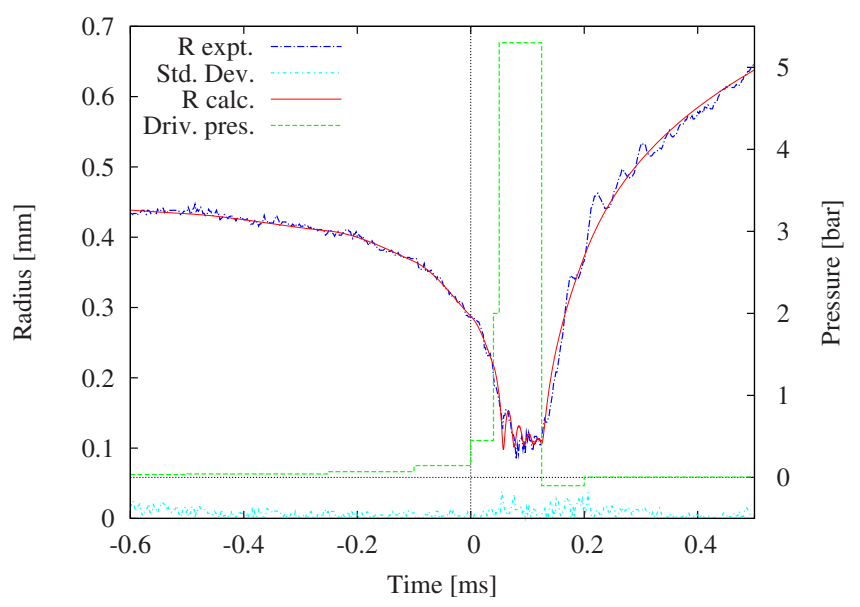

FIG. 8. (Color online) Average of experimental bubble radius obtained from Mie scattering technique $\left(R_{\text {exp }}\right)$, its standard deviation (Std. Dev.), stepwise driving pressure approximation (Driv. Pres.) and calculated radius using this pressure approximation $\left(R_{\text {calc }}\right)$ for the first collapse.

collapses. It will also give an estimation for the conditions inside the bubble which result in the light emission. Although this inverse problem may be difficult to solve in general, the approach taken here is simplified by assuming that the pressure evolution we are computing is stepwise constant since, ideally, the water hammer effect produces a discontinuous pressure pulse which has a stepwise shape. Thus we used the following strategy. We initialized the code with bubble conditions a few milliseconds before the collapse. We provided a time interval and looked for the constant pressure that best fitted bubble radius evolution over that time interval. Evidently, the approximation is improved as the time interval is refined. Then we refined the time interval until a prescribed level of accuracy was achieved (not necessarily the same for different time intervals). The time interval had to be long enough to keep a reasonable amount of experimental points within the time interval. In addition, we are not interested in recovering small time scale radius fluctuations (especially seen after collapses, in Figs. 8 and 9). Once the

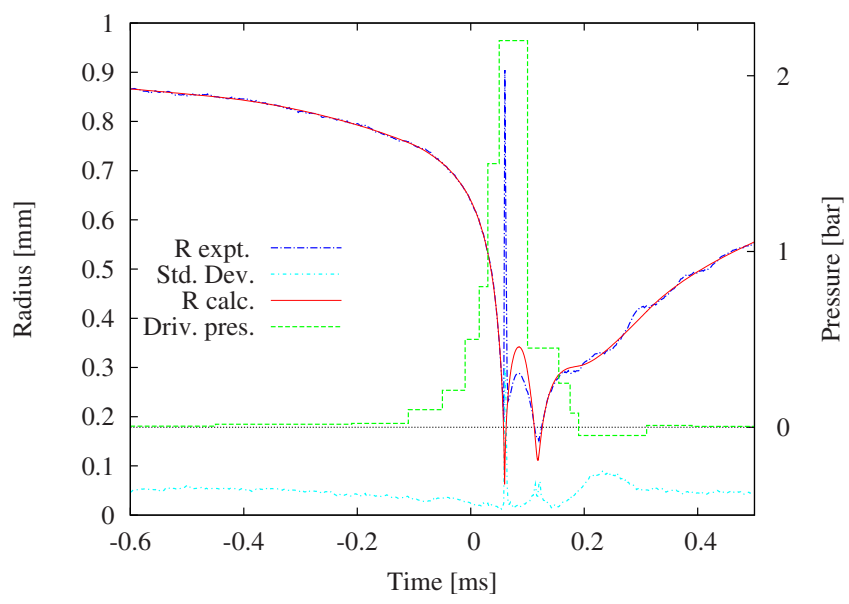

FIG. 9. (Color online) Same data of Fig. 8 corresponding to the second collapse for the upper bubble. pressure and the time interval were obtained the process was repeated giving the next pressure and time interval and so on. Results from this process applied to the first and second collapse of the upper bubble are shown in Figs. 8 and 9, respectively.

\section{B. Numerical results}

Figure 8 shows the average of six experimental radius determinations for the first collapse ( $R_{\exp }$ curve) obtained in similar experimental conditions than that of Fig. 7, along with the standard deviation of the set ("Std. Dev." curve). This magnitude serves as the experimental variance estimation and its low value indicates that the process is repetitive. It is also shown the stepwise approximation obtained for the driving pressure ("Driv. Pres." curve), and the fitted radius evolution $\left(R_{\text {calc }}\right.$ curve) resulting from this stepwise driving pressure. The computation was initialized with bubble conditions at $3.4 \mathrm{~ms}$ before the first collapse (see Fig. 7 which shows one single determination, while $R_{\text {exp }}$ curve in Fig. 8 is the average of six determinations). In that moment the radius is about $0.46 \mathrm{~mm}$, approximately constant, the gas temperature is taken equal to the liquid temperature $(297 \mathrm{~K})$ and the gas pressure is taken to be 27 mbar (free surface pressure) plus the pressure jump due to surface tension. These parameters determine the amount of gas (xenon) inside the bubble.

The pressure profile obtained (Fig. 8) resembles the ideal discontinuous pulse expected in that its height agrees with a priori estimations (6 bar, from liquid column velocity, Fig. 5) and its characteristic time of variation also agrees with estimations since pressure pulse arrives at upper bubble $d_{\mathrm{UB}} / c_{l}$ $\sim 60 \mu$ s (where $d_{\mathrm{UB}} \sim 8 \mathrm{~cm}$ is the distance from tube bottom to upper bubble, and $c_{l}=1400 \mathrm{~m} / \mathrm{s}$ ) after the cavity collapse is detected (indicated by $t=0$ in Fig. 8) and pressure pulse duration is of the same order of magnitude. Bubble radius presented in Fig. 8 shows that bubble compression begins before the cavity collapse. This motion is associated with a gradual pressure increase preceding the main pulse in the stepwise pressure approximation and it provides information about how the compression over the liquid column is produced by the collapsing cavity. This gradual pressure increase before the main pulse diminishes the bubble size before the moment of maximum compression making the collapse less severe. Negative pressures are found behind the main pressure pulse, followed by a low pressure instance ( $\sim 5.5$ mbar) which remains until approximately $500 \mu$ s before the second collapse. Negative pressures were expected since compression wave launched by the cavity collapse reflects at the column free surface as a tensile wave which then produces negative pressure at the bottom of the tube. This process results in a bubble growth after the first collapse reaching a bubble radius of almost $1 \mathrm{~mm}$ (Fig. 7) and setting up favorable conditions for a more violent second collapse.

Measurements (without the red filter used in measurements presented in Fig. 7) and calculation of second collapse for the upper bubble are presented in Fig. 9. In this case two experimental radius evolutions were averaged. Bubble conditions at $1 \mathrm{~ms}$ before the second collapse (indicated in Fig. 7) were used as initial conditions for the computation. Initial 
temperature is again liquid temperature and the gas pressure is deduced from the amount of xenon which is held constant from the first collapse calculation. Resulting stepwise driving pressure in this case is, as expected, weaker (2.2 bar maximum) and so radius fluctuations following collapse, but typical times of time intervals for pressure variation do not change significantly. In spite of weaker driving, second collapse is seen to be more violent and extreme conditions resulting from both calculations confirm that since the maximum temperature reached in first collapse is below $2000 \mathrm{~K}$ (not enough for visible light emission), while the second collapse maximum temperature is about $11500 \mathrm{~K}$ with 1.4 $\times 10^{11}$ photons emitted per pulse (the corresponding total peak power emitted is $0.38 \mathrm{~W}$ ) during $160 \mathrm{~ns}$ (FWHM). Measured light emission for collapses similar to that showed in Fig. 9 gave $2 \times 10^{11}$ photons per pulse and $250 \pm 50 \mathrm{~ns}$ of FWHM $(t=260 \mathrm{~s}$ in Fig. 3). These values are in the same order of magnitude that previously reported data [11]. When a bubble subjected to a constant liquid pressure collapses, the mechanical energy transferred into the bubble is proportional to the liquid pressure and the initial bubble volume [14]. Thus, one may conclude that the second collapse is more violent than the first one simply because the initial radius of the collapsing bubble is larger. Nevertheless, the collapses undertaken by the upper bubble, Figs. 8 and 9, are not subjected to a constant driving pressure. In particular, the gradual pressure increase observed before the main pulse produces a significant bubble volume reduction that prevents the main pulse from transferring more mechanical energy into the bubble. This phenomenon is clearly seen in Fig. 8, where main pressure pulse arrives when the bubble is almost at its minimum radius. In the second collapse, Fig. 9, the main pressure pulse is smaller but it has better chances for energy transmission because it acts when bubble is larger. Therefore, we conclude that the second collapse is more violent and leads to light emission not only because it has a larger initial radius but also because the lower driving pressure is more efficient in energy transmission. In order to analyze this behavior we studied bubble conditions at the collapse as a function of initial radius $\left(R_{\text {ini }}\right)$ and driving pressure strength. Results are shown in Fig. 10. There, contours of temperature, total peak power emitted and FWHM resulting from the numerical model are traced in maximum driving pressure versus initial radius space. Each point of this space is associated with a bubble collapse beginning from a particular initial radius ( $x$ axis) and forced by the stepwise pressure profile of Fig. 9 multiplied by a constant ( $y$ axis, where the labels indicate maximum driving pressure).

The filled point in Fig. 10 shows the position of the second collapse $\left(R_{\mathrm{ini}}=0.89 \mathrm{~mm}, P_{\max }=2.2 \mathrm{bar}\right)$, and the empty point the first one $\left(R_{\text {ini }}=0.46 \mathrm{~mm}, P_{\max }=5.3 \mathrm{bar}\right)$. It is seen that the numerical results agree with the experimental observation that light emission is detected in the second collapse but not in the first one. It is also observed that, in general, increasing initial radius leads to more intense collapses (in terms of temperature and light emission) as in the constant pressure collapse case. In contrast, the effect of increasing driving pressure is not so straightforward. Keeping $R_{\text {ini }}$ constant, it is observed that pressure intensity presents an optimum value that produces the brightest collapses (about a

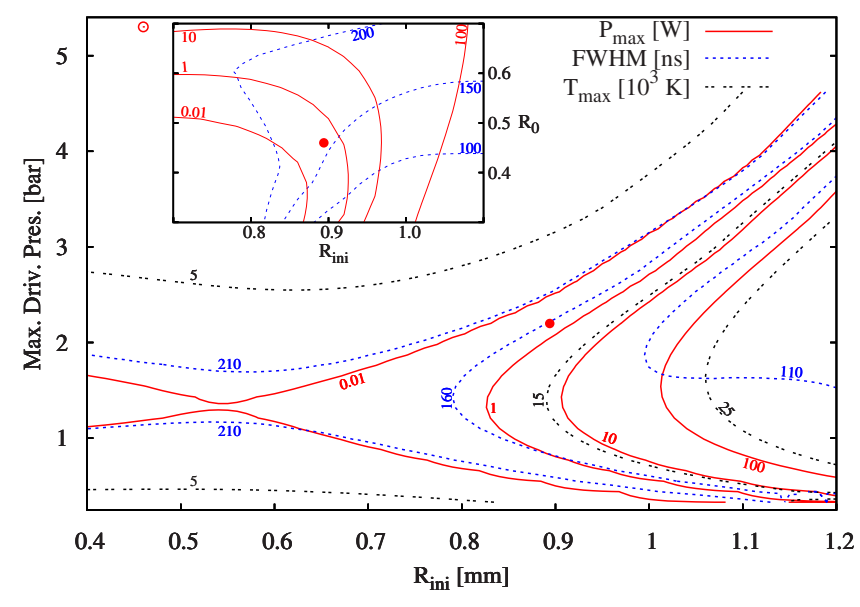

FIG. 10. (Color online) Isolines of calculated total peak power emitted $\left(P_{\max }\right)$, FWHM, and temperature in maximum driving pressure-initial radius space. The inset shows contours of total peak power emitted and FWHM when the amount of gas inside the bubble is varied $\left(R_{0}\right.$ vs $\left.R_{\text {ini }}\right)$ while maximum driving pressured is held constant (at 2.2 bar).

maximum pressure of 1.4 bar). Note that if the driving pressure of the first collapse is applied to a bubble with the initial radius of the second collapse it will result in a weaker collapse without light emission [maximum temperature below $5000 \mathrm{~K}$, point $(0.89 \mathrm{~mm}, 5.3 \mathrm{bar})$ in Fig. 10]. On the other hand, a bubble with the initial radius of the first collapse can emit some light if a weaker driving is applied [point (0.46 mm, 1.4 bar) in Fig. 10]. Therefore, we conclude that the weaker collapse of the bottom cavity which imposes a weaker driving pressure in the second collapse plays a key role in liquid hammer light emission. We checked that the graph of Fig. 10 does not change significantly and the above discussion is not modified when the stepwise pressure approximation of Fig. 8 is used instead of the pressure approximation of Fig. 9 (using appropriate multiplicative factors in order to obtain the same maximum pressures, i.e., $y$-axis range). Calculations discussed up to this point involved the same amount of gas inside the bubble. We specified this quantity to be the amount of gas that makes a bubble of $0.46 \mathrm{~mm}\left(R_{0}\right)$ at room temperature have a pressure of 27 mbar (plus surface tension). We note that this somewhat arbitrary value may change during experiment evolution due to rectified diffusion [14] and coalescence with small bubbles which come from lower positions in the tube. The effect of varying the amount of gas inside the bubble, on light emission is showed in the inset of Fig. 10. Contours of total peak power emitted and pulse width (FWHM) are traced in $R_{0}$ vs $P_{\text {max }}$ space. Initial radius for these computations was taken equal to $0.89 \mathrm{~mm}$ as in the second collapse. We observe that as the bubble grows the pulses emitted become brighter and wider, which is in agreement with experimental observations (Fig. 3). We also note that a sudden increase in pressure amplitude (i.e., at constant $R_{0}$ ) would diminish the amount of light emitted. This fact is also in agreement with the experiment since, as discussed in the context of Fig. 3, we observed that a high increase in tube oscillation amplitude (which leads to an increase of the driving pressure imposed 
by the cavity collapse) tend to diminish the intensity of the light emitted by the upper bubble. On the other hand, we found that an oscillation amplitude increase (and consequently a driving pressure increase) can cause the upper bubble to emit more light when it is applied gradually, i.e. after the bubble has grown enough (increasing its $R_{0}$ ), as shown in the inset of Fig. 10. In this way, pulses up to 2 $\times 10^{13}$ photons (20 W peak power) were achieved after $40 \mathrm{~min}$ of operation.

\section{CONCLUSIONS}

The collapse of the cavity at the bottom of the tube results in the sudden compression of the liquid column. This compression is 100 times higher than gas pressure filling the tube and drives impulsively the dynamics of the bubbles placed along the liquid column. Upper sonoluminescing bubble undergoes a series of collapses synchronized with liquid column compressions. In each excitation cycle, light emission takes place at the second collapse of the upper bubble in spite of the fact that the first collapse is driven by a higher pressure. Such behavior is a consequence of pressure driving profile imposed in the liquid column by the cavity flow. This pressure driving profile presents a gradual pressure increase preceding the main compression that reduces significantly bubble volume, diminishing the mechanical energy transfer into the bubble from the main pressure pulse. For a given initial radius $\left(R_{\text {ini }}\right)$ there is an optimal pressure pulse amplitude that leads the maximum light emission. The numerical calculations are in quantitative agreement with observations of radius and light emission (over the intensity as well as the temporal width). They also reproduce the observation of pulses becoming brighter and wider as experiment time advances, when gas incorporation into the upper bubbles is taken into account. We emphasize that although the number of photon emitted per pulse in this experiment is about five orders of magnitude greater than SL produced in water, the predicted maximum temperatures inside the bubble are below $20000 \mathrm{~K}$ (similar or even lower than in SL in water). Finally, we want to mention that conditions inside the inferior cavity at its main collapse may be more extreme, in terms of maximum temperature achieved. In this case, the light pulses are less intense and shorter than light pulses from upper bubble. Nevertheless, temperature estimations in this case are very difficult because of the asymmetry introduced by the tube wall.

\section{ACKNOWLEDGMENTS}

We acknowledge the technical support of Enrique Aburto, Sebastián Eckardt, and Daniel Mateos. R.U. and P.L.G.M. were financed by CONICET/CNEA.
[1] D. F. Gaitán and L. A. Crum, Frontiers of Nonlinear Acoustics. 12th ISNA (Elsevier, London, 1990).

[2] M. P. Brenner, S. Hilgenfeldt, and D. Lohse, Rev. Mod. Phys. 74, 425 (2002).

[3] S. Hilgenfeldt and D. Lohse, Phys. Rev. Lett. 82, 1036 (1999).

[4] J. Holzfuss, M. Ruggeberg, and R. Mettin, Phys. Rev. Lett. 81, 1961 (1998).

[5] R. Toegel, B. Gompf, R. Pecha, and D. Lohse, Phys. Rev. Lett. 85, 3165 (2000).

[6] D. J. Flannigan and K. S. Suslick, Nature (London) 434, 52 (2005).

[7] G. F. Puente, P. García-Martínez, and F. J. Bonetto, Phys. Rev. E 75, 016314 (2007).

[8] R. Urteaga, D. H. Dellavale, G. F. Puente, and F. J. Bonetto, Phys. Rev. E 76, 056317 (2007).
[9] R. Urteaga and F. J. Bonetto, Phys. Rev. Lett. 100, 074302 (2008).

[10] C. K. Su, C. Camara, B. Kappus, and S. J. Putterman, Phys. Fluids 15, 1457 (2003).

[11] A. Chakravarty, T. Georghiou, T. E. Phillipson, and A. J. Walton, Phys. Rev. E 69, 066317 (2004).

[12] B. P. Barber and S. J. Putterman, Phys. Rev. Lett. 69, 3839 (1992)

[13] B. Gompf and R. Pecha, Phys. Rev. E 61, 5253 (2000).

[14] T. Leighton, The Acoustic Bubble (Academic, London, 1994).

[15] S. Hilgenfeldt, S. Grossmann, and D. Lohse, Phys. Fluids 11, 1318 (1999).

[16] Rayleigh collapse time for a $0.8 \mathrm{~mm}$ radius bubble at 1 bar is smaller than $100 \mu \mathrm{s}$, in phosphoric acid. 